\title{
- The associated production of vector bosons and jets 2 originating from heavy-flavour quarks and constrain on 3 PDFs in CMS
}

\author{
4 Anton Stepennov on behalf of the CMS Collaboration. ${ }^{a, *}$ \\ $5{ }^{a}$ Institute for Theoretical and Experimental Physics, \\ 6 Moscow, Russia \\ $7 \quad$ E-mail: anton.stepennov@cern.ch
}

The associated production of vector bosons V (W, Z or gamma) and jets originating from heavyflavour (c or b) quarks is a large background source in measurements of other standard model processes, Higgs boson studies, and many searches for physics beyond the standard model. The study of events with a vector boson accompanied by heavy-flavour jets is crucial to refine the theoretical calculations in perturbative QCD, as well as to validate associated Monte Carlo predictions. Differential cross sections in $\mathrm{V}+\mathrm{c} / \mathrm{b}$ jets are measured as a function of several kinematic observables with the CMS detector at 8 and $13 \mathrm{TeV}$. The study of the associated production of a vector boson with jets from a c-quark is especially interesting, as it allows to extract information on the proton parton density functions.

40th International Conference on High Energy physics - ICHEP2020

July 28 - August 6, 2020

Prague, Czech Republic (virtual meeting)

\footnotetext{
${ }^{*}$ Speaker
} 


\section{Introduction}

The CMS experiment [1] allows a wide range of measurements of events with vector bosons and jets originating from heavy-flavour quarks. Studies of the associated production of a $\mathrm{W}$ boson with a charm quark at 8 an $13 \mathrm{TeV}$, and the associated production of a $\mathrm{Z}$ boson with charm or bottom quarks at $13 \mathrm{TeV}$ are presented in this work.

\section{W bosons with charm quarks at $13 \mathrm{TeV}$}

The associated production of a $\mathrm{W}$ boson and a charm quark is sensitive to the strange component of the proton due to the leading order (LO) processes $\mathrm{g}+\mathrm{s} \rightarrow \mathrm{W}^{-}+\mathrm{c}$ and $\mathrm{g}+\overline{\mathrm{s}} \rightarrow \mathrm{W}^{+}+\overline{\mathrm{c}}$.

The study of a $\mathrm{W}$ boson and a charm quark at $13 \mathrm{TeV}$ [2] is done using events with a $\mathrm{W}$ boson, decaying into muon and neutrino, and a c quark, hadronized into a $D^{*}(2010)^{ \pm}$meson. W boson candidates are selected by requiring isolated muons with $p_{\mathrm{T}}^{\mu}>26 \mathrm{GeV}$ and $\left|\eta^{\mu}\right|<2.4$. The neutrino from $\mathrm{W}$ boson decay remains undetected, thus its presence can be identified by large missing transverse momentum $p_{\mathrm{T}}^{\text {miss }}>50 \mathrm{GeV}$. The decay chain $D^{*}(2010)^{ \pm} \rightarrow D^{0}+\pi^{ \pm}$slow $\rightarrow$ $K^{\mp}+\pi^{ \pm}+\pi^{ \pm}$slow is used to reconstruct $D^{*}(2010)^{ \pm}$mesons.

Selected events with $\mathrm{W}$ bosons and $D^{*}$ mesons are divided into two categories: same sign (SS) $W^{ \pm}+D^{*}(2010)^{ \pm}$and opposite sign (OS) $W^{ \pm}+D^{*}(2010)^{\mp}$. By subtracting SS from OS candidates, the symmetric background events are drastically reduced.

It can be shown in the simulation, that the requirement $p_{\mathrm{T}}^{D^{*}}>5 \mathrm{GeV}$ translates into $p_{\mathrm{T}}^{c}>5$ $\mathrm{GeV}$, so the fiducial cross-section volume is defined as $p_{\mathrm{T}}^{c}>5 \mathrm{GeV}, p_{\mathrm{T}}^{\mu}>26 \mathrm{GeV}$ and $\left|\eta^{\mu}\right|<2.4$. The fiducial cross section is found to be $1026 \pm 31$ (stat) ${ }_{-72}^{+76}$ (syst) pb, while the measured charge asymmetry $\sigma\left(W^{+}+\bar{c}\right) / \sigma\left(W^{-}+c\right)$ equals $0.968 \pm 0.055$ (stat) $)_{-0.028}^{+0.015}$ (syst) .

The fiducial cross section for $\mathrm{W}+D^{*}(2010)^{\mp}$ can also be measured, allowing to reduce uncertainties, related to interpolation to the $\mathrm{W}+\mathrm{c}$ region. It is found to be $190 \pm 6(\mathrm{stat})_{-13}^{+12}(\mathrm{syst})$ $\mathrm{pb}$, with charge asymmetry equal to $0.909 \pm 0.051$ (stat) ${ }_{-0.028}^{+0.014}$ (syst).

Differential cross sections for $\mathrm{W}+\mathrm{c}$ and $\mathrm{W}+D^{*}(2010)^{ \pm}$measured as functions of $\left|\eta^{\mu}\right|$ are shown in Fig. 1.

Both inclusive and differential cross-sections are compared with theoretical predictions using different PDF sets and all except ATLASepWZ16nnlo [3] agree well with the measurements. Results of differential cross section measurement for $\mathrm{W}+D^{*}(2010)^{ \pm}$are in good agreement with the predictions of MadGraph5_aMC@NLO [4].

The differential $\mathrm{W}+\mathrm{c}$ cross section is also sensitive to the PDF of the strange quark. The $\mathrm{W}+\mathrm{c}$ production cross sections presented here, together with HERA inclusive cross sections [5] and other CMS $\mathrm{W}$ and $\mathrm{W}+\mathrm{c}$ measurements [6-8] were used in an NLO QCD analysis. Its results for s quark and the strangeness suppression factor $r\left(x, \mu_{F}^{2}\right)=(s+\bar{s}) /(\bar{u}+\bar{d})$ are shown in Fig. 2. (left) for factorization scale $\mu_{F}^{2}=M_{W}^{2}$. Fig. 2. (right) shows a comparison of the strangeness suppression factor with ATLASepWZ16 and ABMP16 fits. The CMS result is close to ABMP16 and agrees with earlier results from neutrino scattering experiments. It does not support the hypothesis of an enhanced strange quark contribution in the proton quark sea reported by ATLAS [3]. 

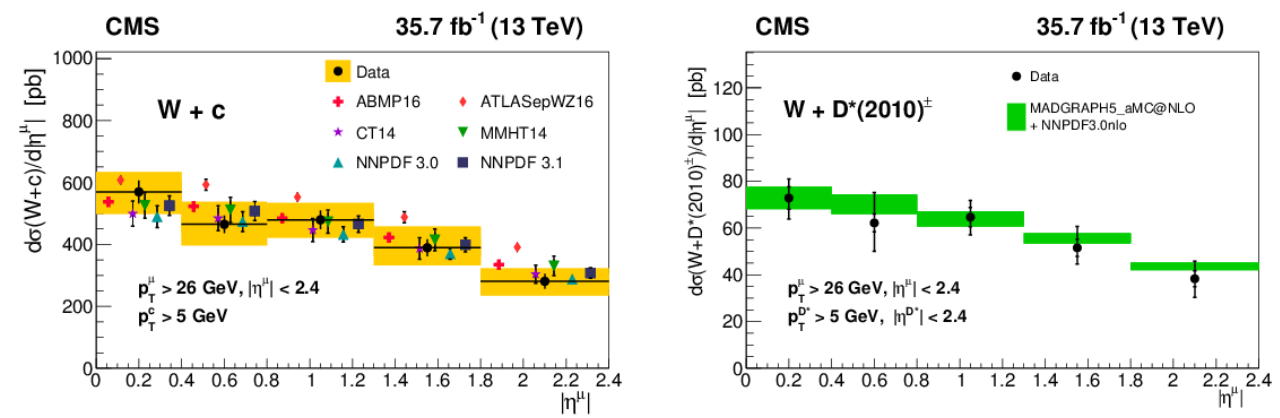

Figure 1: Differential cross sections $\mathrm{W}+\mathrm{c}$ (left) and $\mathrm{W}+D^{*}(2010)^{ \pm}$(right) at $13 \mathrm{TeV}[2]$.
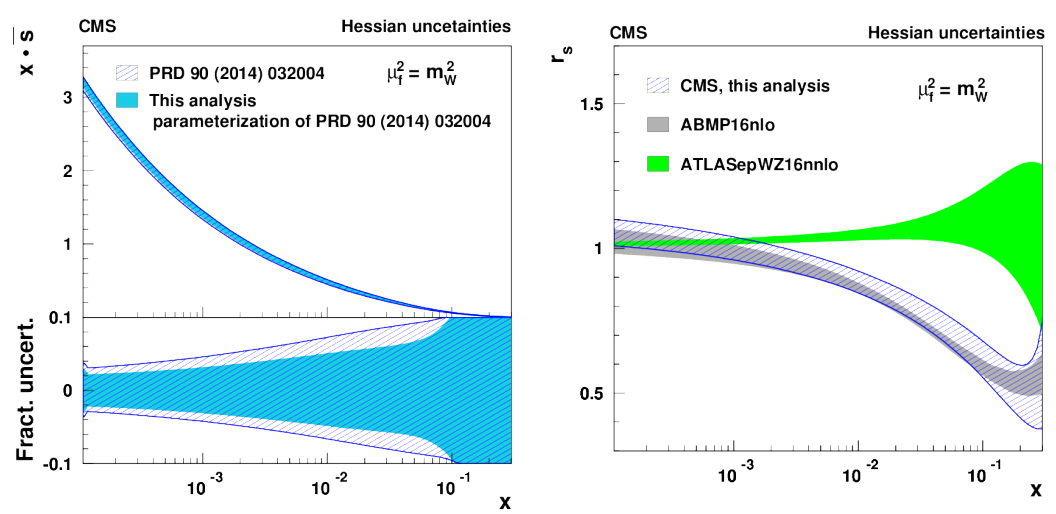

Figure 2: Distribution of s quark and strangeness suppression factor [2].

\section{W bosons with charm quarks at $8 \mathrm{TeV}$}

For the measurement of associated production of a $\mathrm{W}$ boson with a charm quark at $8 \mathrm{TeV}$ [9] W bosons decaying into electron or muon are considered, along with hadronic jets, originating from a c quark (c jets). W bosons candidates are selected by requiring isolated muons or electrons with $p_{\mathrm{T}}$ greater than $30 \mathrm{GeV}$ and $|\eta|<2.1$. In order to take into account neutrinos from decay of $\mathrm{W}$ boson, the cut for transverse mass is applied $m_{T}>55 \mathrm{GeV}$. Jets are selected with $p_{\mathrm{T}}^{\text {jet }}>25 \mathrm{GeV}$ and $\left|\eta^{j e t}\right|<2.5$, separated from leptons from W boson decay $\Delta R($ jet,$\ell)>0.5$.

The $\mathrm{c}$ jets are selected in two different channels. The first one is semileptonic channel, with an identified muon inside the selected jet, with $p_{\mathrm{T}}^{\mu}<25 \mathrm{GeV}, p_{\mathrm{T}}^{\mu} / p_{\mathrm{T}}^{j e t}<0.2$ and $\left|\eta^{\mu}\right|<2.1$. Unlike leptons from $\mathrm{W}$ boson decays, this muon shouldn't be isolated from hadronic activity.

In the second channel jets are required to contain a reconstructed secondary vertex (SV), displaced from the primary vertex (PV). The invariant mass of the tracks, associated with this SV is required to be greater, than $0.55 \mathrm{GeV}$. The sign of the charm hadron is determined by the total charge of the tracks, associated with the SV or, in case if the charge is equal to zero, the charge of the track from the PV, closest to the $\mathrm{SV}$, is chosen.

After combining different channels the fiducial cross section times $\mathrm{W}$ boson leptonic branching equals $116.3 \pm 0.7$ (stat) \pm 5.2 (syst) pb and charge asymmetry is found to be $0.986 \pm 0.011$ (stat) \pm 0.013(syst). Differential cross section and charge asymmetry as a function of selected lepton pT are shown in Fig. 3. These results are compared with predictions of the MCFM program, using 4 
different PDF sets, and MadGraph5_aMC@NLO v5.1.3.30 LO MC simulation. MCFM tends to slightly underestimate data, while there is a good agreement with MadGraph5_aMC@NLO.
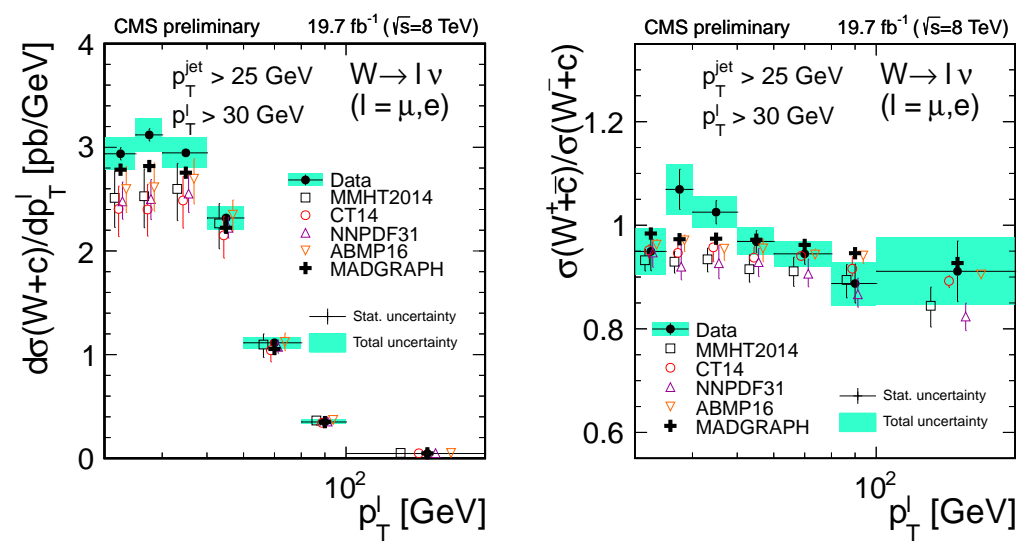

Figure 3: Differential cross section (left) and charge asymmetry (right) $\mathrm{W}+\mathrm{c}$ at $8 \mathrm{TeV}$ [9] as function of lepton $p_{\mathrm{T}}$.

\section{4. $\mathrm{Z}$ bosons with charm quarks at $13 \mathrm{TeV}$}

The measurement of the associated production of a $\mathrm{Z}$ boson with charm quarks allows to test existing MC models and can improve constraints on the charm quark distribution in the proton.

In this study at $13 \mathrm{TeV}$ [10] the $\mathrm{Z}$ boson is reconstructed from the decays to pair of muons or electrons. These leptons are required to be isolated and pass selection criteria: $p_{\mathrm{T}}^{\ell}>26$ (29) $\mathrm{GeV}$ for muons (electrons), $\left|\eta^{\ell}\right|<2.4,71 \mathrm{GeV}<M_{\ell \ell}<111 \mathrm{GeV}$.

Jets with $p_{\mathrm{T}}^{j}>30 \mathrm{GeV}$ and $\left|\eta^{j}\right|<2.4$ are selected. A sample enriched with $\mathrm{c}$ jets is selected by applying a $\mathrm{c}$ tagging criteria (c tagged jets) using the deep combined secondary vertices algorithm [11]. This algorithm is based on a deep neural network, which uses different jet parameters, such as mass of SV or number of tracks, associated with the SV.

To make possible a comparison of the measurement results with predictions of different MC generators, the data is unfolded to account for detector effects.

The number of signal events is measured by fitting the SV mass distribution for the selected $\mathrm{c}$ tagged jet, in data and MC. The contribution from background with top quarks or dibosons is small, these events are subtracted from data. The results of such measurement are unfolded using generator level signal definition. The differential cross section as a function of $p_{\mathrm{T}}$ of the selected $\mathrm{Z}$ boson or $\mathrm{c}$ jet are shown in Fig. 4.

The total fiducial cross section is found to be $405.4 \pm 5.6($ stat $) \pm 23.5(\exp ) \pm 8.1$ (th) pb. These results are compared with predictions of three MC generators: MadGraph5_aMC@NLO at leading order, MadGraph5_aMC@NLO at next-to-leading order and Sherpa [12] at next-to-leading order. All three models predictions were normalized to the cross section, calculated at next-to-next-toleading order. The predictions at next-to-leading order by Sherpa and MadGraph5_aMC@NLO tend to overestimate the data, while there is good a agreement with MadGraph5_aMC@NLO at leading order. 

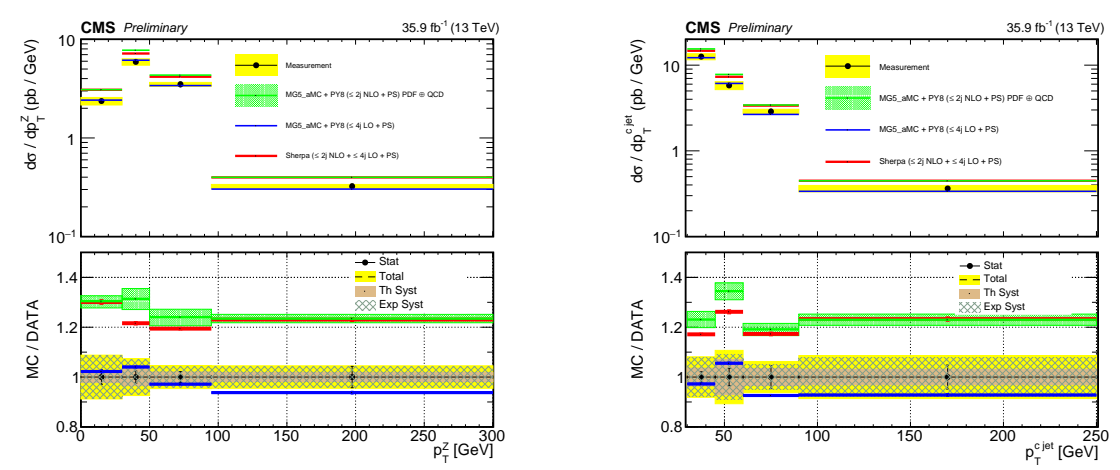

Figure 4: Differential cross section $\mathrm{Z}+\mathrm{c}$ jet at $13 \mathrm{TeV}[10]$, as function of $\mathrm{Z}$ boson $p_{\mathrm{T}}(\mathrm{left})$ and $\mathrm{c}$ jet $p_{\mathrm{T}}$ (right).

\section{Z bosons with charm and bottom quarks at $13 \mathrm{TeV}$}

The measurement of cross sections ratios allows to decrease systematic uncertainties. In this study at $13 \mathrm{TeV}[13]$ three ratios are measured: $\sigma(\mathrm{Z}+\mathrm{c}$ jets $) / \sigma(\mathrm{Z}+$ jets $), \sigma(\mathrm{Z}+\mathrm{b}$ jets $) / \sigma(\mathrm{Z}+$ jets $)$, and $\sigma(\mathrm{Z}+\mathrm{c}$ jets $) / \sigma(\mathrm{Z}+\mathrm{b}$ jets $)$, both for inclusive and differential cross sections. The fiducial volume of this measurement is defined by jet $p_{\mathrm{T}}^{\text {jet }}>30 \mathrm{GeV}, \eta^{\text {jet }}<2.4$, lepton (muon or electron) $p_{\mathrm{T}}^{\ell}>25 \mathrm{GeV}, \eta^{\ell}<2.4$. The $\mathrm{Z}$ boson is selected by requiring the invariant mass of two opposite sign leptons to be in range 71 and $111 \mathrm{GeV}$. The sample is enriched with $\mathrm{c}$ and $\mathrm{b}$ jets by requiring jets to pass deep CSVv2 $b$ tagging criteria [11]. The exact composition of $Z+c$ and $Z+b$ jet components in data is found through the fit of SV mass distribution. In order to take into account detector effects, results of the measurement are unfolded. Fig. 5. shows fractions of $Z+c$ jets and $\mathrm{Z}+\mathrm{b}$ jets as a function of $\mathrm{c}$ or $\mathrm{b}$ jet $p_{\mathrm{T}}$. MadGraph5_aMC@NLO predictions at leading and next-to-leading order tend to slightly overestimate the data.
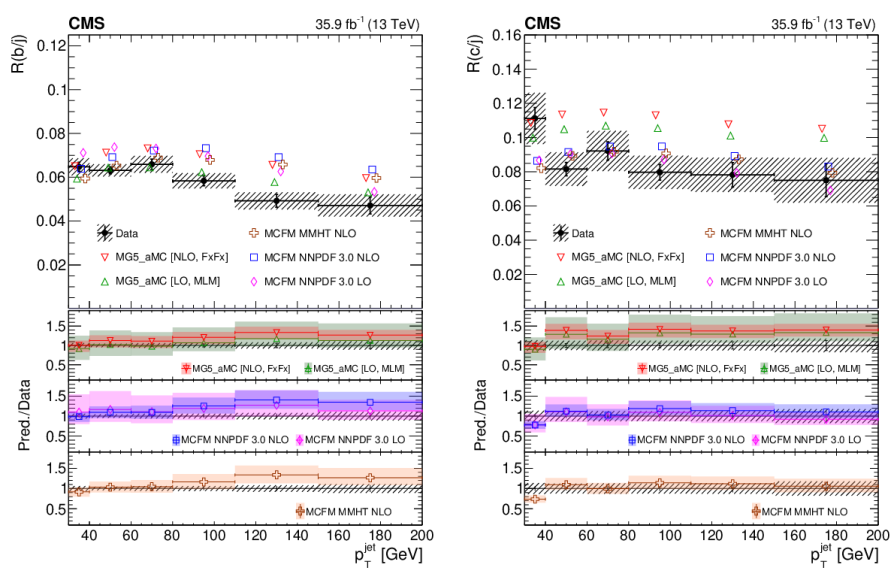

Figure 5: Differential cross sections ratios $(\mathrm{Z}+\mathrm{b}$ jets $) /(\mathrm{Z}+$ jets $)($ left $)$ and $(\mathrm{Z}+\mathrm{c}$ jets $) /(\mathrm{Z}+$ jets $)$ (right) at $13 \mathrm{TeV}$ [13] as function of jet $p_{\mathrm{T}}$. 
0.009 (syst), $\sigma(\mathrm{Z}+\mathrm{b}$ jets $) / \sigma(\mathrm{Z}+$ jets $)=0.0633 \pm 0.0004$ (stat $) \pm 0.0015$ (syst), and $\sigma(\mathrm{Z}+\mathrm{c}$ jets $) / \sigma(\mathrm{Z}+\mathrm{b}$ jets $)$ $=1.62 \pm 0.03$ (stat) \pm 0.15 (syst).

\section{References}

[1] The CMS Collaboration, The CMS Experiment at the CERN LHC, JINST 3 (2008), S08004

[2] The CMS Collaboration, Measurement of associated production of a $W$ boson and a charm quark in proton-proton collisions at $\sqrt{s}=13 \mathrm{TeV}$, Eur. Phys. J. C 79 (2019) no.3, 269

[3] M. Aaboud et al. [ATLAS], Precision measurement and interpretation of inclusive $W^{+}, W^{-}$ and $Z / \gamma^{*}$ production cross sections with the ATLAS detector, Eur. Phys. J. C 77 (2017) no.6, 367

[4] J. Alwall, R. Frederix, S. Frixione, V. Hirschi, F. Maltoni, O. Mattelaer, H. S. Shao, T. Stelzer, P. Torrielli and M. Zaro, The automated computation of tree-level and next-to-leading order differential cross sections, and their matching to parton shower simulations, JHEP 07 (2014), 079

[5] H. Abramowicz et al. [H1 and ZEUS], Combination of measurements of inclusive deep inelastic $e^{ \pm} p$ scattering cross sections and QCD analysis of HERA data, Eur. Phys. J. C 75, no.12, 580 (2015)

[6] The CMS Collaboration, Measurement of the Muon Charge Asymmetry in Inclusive $p p \rightarrow$ $W+X$ Production at $\sqrt{s}=7 \mathrm{TeV}$ and an Improved Determination of Light Parton Distribution Functions, Phys. Rev. D 90, no.3, 032004 (2014)

[7] The CMS Collaboration, Measurement of Associated $W+$ Charm Production in pp Collisions at $\sqrt{s}=7 \mathrm{TeV}$, JHEP 02, 013 (2014)

[8] The CMS Collaboration, Measurement of the differential cross section and charge asymmetry for inclusive $\mathrm{pp} \rightarrow \mathrm{W}^{ \pm}+X$ production at $\sqrt{s}=8 \mathrm{TeV}$, Eur. Phys. J. C 76, no.8, 469 (2016)

[9] The CMS Collaboration, Measurement of the associated production of a W boson and a charm quark at $\sqrt{s}=8 \mathrm{TeV}$, CMS-PAS-SMP-18-013, http://cds. cern. ch/record/2682200

[10] The CMS Collaboration, Differential cross section measurements of the production of Z bosons and charm jets at $\sqrt{s}=13 \mathrm{TeV}$, CMS-PAS-SMP-19-011, http://cds.cern.ch/record/ 2723840

[11] The CMS Collaboration, Identification of heavy-flavour jets with the CMS detector in pp collisions at $13 \mathrm{TeV}$, JINST 13 (2018) no.05, P05011

[12] T. Gleisberg, S. Hoeche, F. Krauss, M. Schonherr, S. Schumann, F. Siegert and J. Winter, Event generation with SHERPA 1.1, JHEP 02 (2009), 007

[13] The CMS Collaboration, Measurement of the associated production of a $Z$ boson with charm or bottom quark jets in proton-proton collisions at $\sqrt{s}=13 \mathrm{TeV}$, Phys. Rev. D 102 (2020) no.3, 032007 